\title{
Suboptimal neoaortic arch geometry correlates with inefficient flow patterns in hypoplastic left heart syndrome
} \author{
Colo
}

Michal Schäfer, PhD, ${ }^{\mathrm{a}}$ Michael V. DiMaria, MD, ${ }^{\mathrm{a}}$ James Jaggers, MD, ${ }^{\mathrm{b}}$ and Max B. Mitchell, MD, ${ }^{\mathrm{b}}$ Aurora,

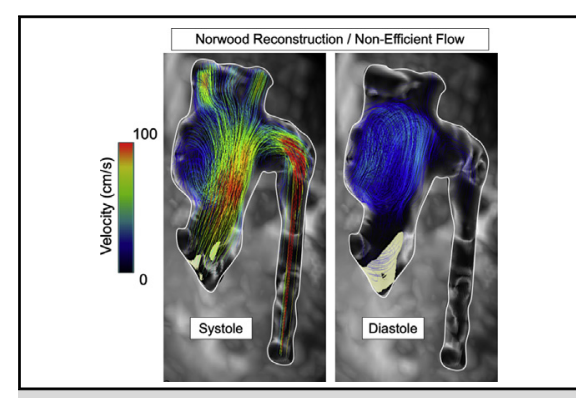

Inefficient flow after Norwood-type aortic arch reconstruction.

Central Message

Norwood-type aortic arch reconstruction can be optimized to improve the efficiency of blood flow conduction by more closely matching the diameter of the native aorta.

See Commentaries on pages e117 and e119. the thoracic aorta, elevate the single ventricle's workload, and impair long-term performance of the single ventricle. In addition, abnormal and chaotic flow may exacerbate neoaortic dilation and further perpetuate the dissipation of kinetic energy provided by the at-risk single ventricle.

Abnormal flow conduction and neoaortic flow energy loss can be assessed with 4-dimensional (4D) flow magnetic resonance imaging (MRI). The purpose of this study was to determine whether reconstructed neoaortic arch morphology in patients with HLHS at differing stages of palliation would correlate with abnormalities of flow propagation and viscous energy loss.

\section{MATERIALS AND METHODS}

Four patients with HLHS who underwent comprehensive cardiac MRI follow-up, including 4D flow MRI evaluation, were selected for size and stage-matched comparative preliminary flow hemodynamic analyses, and paired comparisons were made. All patients underwent neoaortic reconstruction with the Philadelphia technique and complete coarctectomy with interdigitating descending aortic anastomosis. Pair 1 consisted of 2 patients studied before the Fontan operation at age 2 years. Both patients had HLHS, with severe left ventricular hypoplasia, aortic stenosis, and mitral stenosis. Pair 2 consisted of 2 patients studied after the Fontan operation at ages 4 to 5 years. Pair 2 diagnoses were severe left ventricular hypoplasia, aortic atresia, mitral stenosis (patient 2A); and severe left ventricular hypoplasia, aortic stenosis, and mitral stenosis (patient $2 \mathrm{~B}$ ). All patients had undergone first-stage palliation by means of a Norwood procedure with a Sano shunt.

All considered patients underwent a recently adopted single-ventricle evaluation protocol consisting of same-day cardiac MRI, catheterization, and echocardiography. According to catheterization and angiography, there were no residual stenoses, obstructions, or significant pressure gradients.

These findings correlated with Doppler assessment on echocardiography (descending aortic gradient $<10 \mathrm{~mm} \mathrm{Hg}$ in all subjects). All patients underwent 4D flow MRI evaluation of flow hemodynamic patterns, anatomic delineation of the reconstructed aorta, interactive path line visualization, and comparison of the flow-mediated viscous energy loss, calculated as described previously. ${ }^{3}$ Instantaneous viscous energy loss from frictional forces represents the rate of energy dissipation within a predefined region of interest by computation of the viscous component of the Navier-Stokes energy equation for any point in the cardiac cycle. The instantaneous energy loss was quantified for the entire reconstructed aorta along the tract from the level of the neoaortic valve annulus to the middescending aorta. The viscous energy loss calculation was further parceled into 3 distinctive regions: ascending aorta, aortic arch, and descending aorta. Viscous energy loss represents the quantitative approach toward characterization of secondary (chaotic) flow formations typically represented by vortical or helical formations and can be translated to the overall ventricular mechanical power loss.

\section{RESULTS}

At the time of the cardiac MRI evaluation, all patients had moderate right ventricular hypertrophy with good systolic function and no clinical signs of Fontan failure. Patient 2B (after Fontan operation with inefficient flow) had moderate tricuspid valve regurgitation. All other patients had trivial to mild tricuspid valve regurgitation. The forward cardiac outputs in patients $2 \mathrm{~A}$ and $2 \mathrm{~B}$ were similar; therefore, the presence of moderate tricuspid regurgitation in patient $2 \mathrm{~B}$ had no effect on the magnitude of viscous energy loss, as measured in the neoaortic reconstruction under the measured conditions. 
The comparison of 4D flow MRI findings in patients before the Fontan operation is summarized in Figure 1. Patient $1 \mathrm{~A}$ had comparatively efficient flow transduction and obviously more favorable neoaortic geometry, with no apparent dilation or segmental mismatch. Flow in patient 1A had cohesive streamlines throughout systole with no recirculation in diastole. The viscous energy loss profile revealed minimal flow-mediated energy loss, suggesting absence of vortical or helical flow. Patient 1B had obviously suboptimal neoaortic geometry, with a dilated ascending neoaorta and size mismatch between the ascending neoaorta and aortic arch. Patient 1B exhibited a large-scale
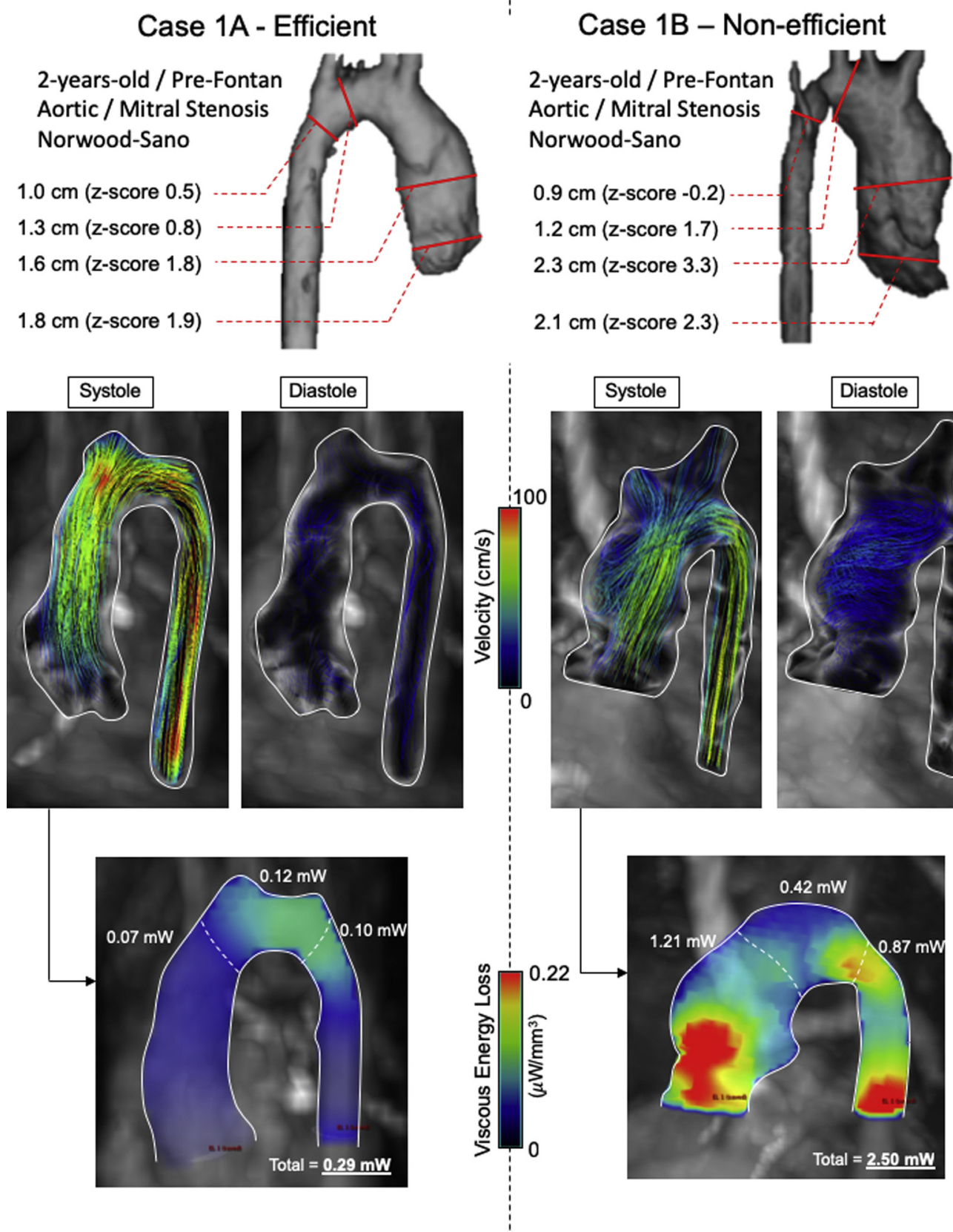

FIGURE 1. Comparison of flow hemodynamic patterns at the pre-Fontan stage of hypoplastic left heart syndrome palliation. In comparison with patient $1 \mathrm{~A}$, patient $1 \mathrm{~B}$ demonstrated a large-scale recirculating vortex in the ascending neoaorta during systole and larger viscous energy loss. The viscous energy loss calculation was further parceled into 3 distinctive regions: ascending aorta, aortic arch, and descending aorta. The viscous energy loss visualization is depicted at peak systole. Viscous energy loss in patient 1B was higher in each aortic region. In addition, patient 1B demonstrated diastolic blood pooling and recirculation in the neo-ascending aorta. 
recirculating vortex in the ascending neoaorta during systole and significantly larger viscous energy loss. In addition, patient 1B exhibited diastolic blood pooling and recirculation in the ascending neoaorta.

The comparison of patients after the Fontan operation is summarized in Figure 2. Patient 2A had comparatively favorable neoaortic size and geometry. Systolic velocity streamlines were cohesive, with laminar flow and no diastolic flow recirculation. Increased viscous energy loss was associated only with the region of elevated flow velocity. Patient 2B had a disproportionally dilated and irregular aorta associated with a large-scale helical flow pattern propagated along the outer curve of the ascending neoaorta. The corresponding viscous energy loss heat map revealed
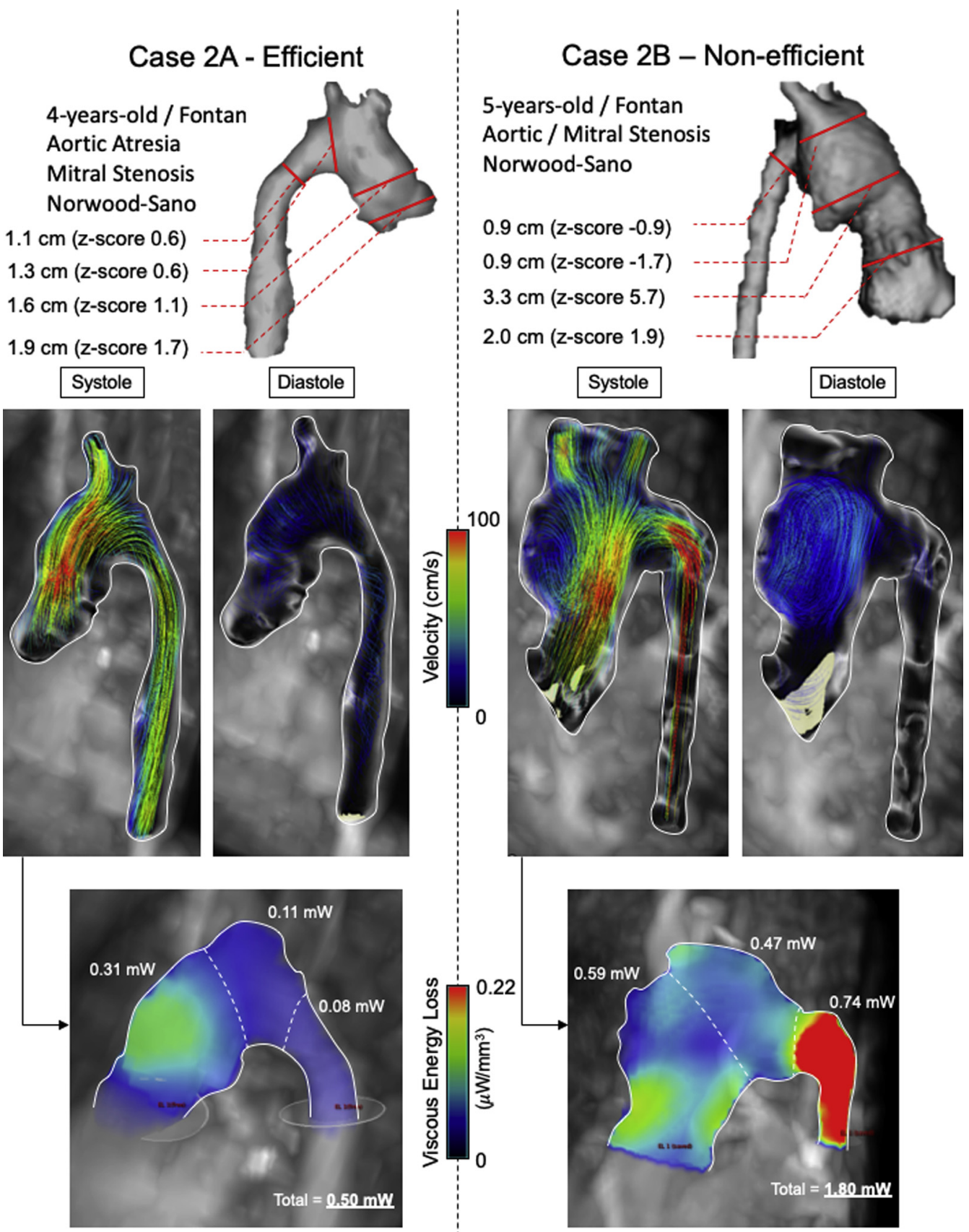

FIGURE 2. Comparison of flow hemodynamic patterns at the post-Fontan stage of hypoplastic left heart syndrome palliation. All flow hemodynamic markers are depicted at peak systole. Patient 2B had a disproportionally dilated and irregular aorta associated with a large-scale helical flow pattern throughout systole, which propagated along the outer curve of the ascending neoaorta. Furthermore, Patient $2 \mathrm{~B}$ demonstrated diastolic recirculation focused within the ascending aorta. Patient 2B further exhibited increased viscous energy loss in each considered aortic segment. 
comparatively high energy loss throughout the entire thoracic aorta. Patient 2B also exhibited diastolic recirculation in the ascending neoaorta.

\section{DISCUSSION}

Flow hemodynamic patterns in the great vessels are predominantly dictated by anatomic shape and the geometry of the ventricular outflow tracts. ${ }^{4,5}$ The geometry for optimal neoaortic flow transmission appears to occur when the shape mirrors that of the normal aorta. This ideal is most closely achieved when the diameter of the proximal ascending neoaorta approximates that of the amalgamation at the native main pulmonary artery and ascending aorta just distal to the pulmonary valve commissures, with a smooth taper to the diameter of the native descending aorta at the distal reconstruction. Excessive dilation of the ascending neoaorta appears to be related to (1) systolic vortex formation along the outer curve of the ascending aorta and (2) diastolic blood pooling in the ascending neoaorta in the form of a recirculating vortex. In addition, viscous energy loss appears to correlate with neoaortic dilation and represents irreversibly wasted energy generated by the single right ventricle. A larger, prospective study is needed to assess these effects on single-ventricle afterload and to associate these factors with long-term outcomes in HLHS. Largescale flow hemodynamic mapping of patients who have undergo stage I Norwood reconstruction will permit development of comprehensive flow hemodynamic atlases and a better understanding of aortic remodeling in this patient population. Surgical efforts to achieve optimal neoaortic geometry consistently could then be assessed and might improve long-term single-ventricle performance. Finally, 4D flow MRI assessments in patients with HLHS may provide clinically useful qualitative and quantitative indices that are more sensitive to detecting flow inefficiencies than are conventional echocardiographic or catheterization techniques.

\section{References}

1. Haller C, Chetan D, Saedi A, Parker R, Van Arsdell GS, Honjo O. Geometry and growth of the reconstructed aorta in patients with hypoplastic left heart syndrome and variants. J Thorac Cardiovasc Surg. 2017;153:1479-87.e1.

2. Bruse JL, Cervi E, McLeod K, Biglino G, Sermesant M, Pennec X, et al; Modeling of Congenital Hearts Alliance (MOCHA) Collaborative Group. Looks do matter! Aortic arch shape after hypoplastic left heart syndrome palliation correlates with cavopulmonary outcomes. Ann Thorac Surg. 2017;103:645-54.

3. Barker AJ, van Ooij P, Bandi K, Garcia J, Albaghdadi M, McCarthy P, et al. Viscous energy loss in the presence of abnormal aortic flow. Magn Reson Med. 2014:72:620-8.

4. Schäfer M, Barker AJ, Kheyfets V, Stenmark KR, Crapo J, Yeager ME, et al. Helicity and vorticity of pulmonary arterial flow in patients with pulmonary hypertension: quantitative analysis of flow formations. J Am Heart Assoc. 2017;6:e007010.

5. Mahadevia R, Barker AJ, Schnell S, Entezari P, Kansal P, Fedak PW, et al. Bicuspid aortic cusp fusion morphology alters aortic three-dimensional outflow patterns, wall shear stress, and expression of aortopathy. Circulation. 2014;129: 673-82. 